\title{
Online efficiency diagnostic of three phase asynchronous machines from start up data
}

\author{
J. Bacher ${ }^{1}, \mathrm{~F}$. Waldhart ${ }^{1}$ \\ ${ }^{1}$ Institute of Drive Technology and Electrical Machines \\ E.A.M, University of Technology Graz \\ Kopernikusgasse 24, 8010 Graz (Austria) \\ Phone/Fax number: +43 316873 8601/10 8601 \\ e-mail: johann.bacher@tugraz.at, waldhart@sbox.tugraz.at
}

\begin{abstract}
In this paper a real time efficiency determination of three phase asynchronous machines is described. Such an online tool improves the reliability of the machines and its maintenance costs. There are various methods to determine the efficiency of three phase induction machines. But all of them have in common that they are to inaccurate or they are impracticable.

Here, the machine efficiency will be derived from start up data. For that purpose the variation with time of all stator currents and all stator voltages have to been measured. No additional data and no additional measuring devices, such rotor speed or torque measurement shaft are needed.
\end{abstract}

\section{Key words}

Three phase induction machine, online efficiency monitoring, start up data, maintenance costs.

\section{Introduction}

Nowadays a cost effective operation of three phase induction machines is absolutely necessary to save energy and maintenance costs. Normally, the load of induction machines is not only constant but also varies within no load and full load. To a minimum of costs the efficiency of the induction machine must be known at each time.

For this reason a simply method to determine the machine efficiency is needed. In [1] fifteen motor efficiency methods are described. The comparison of these methods shows that only two of them are useable because of the error limits. The others such as the power rating plate are unusable.

The comparison of the methods shows very plainly that there is a rampant ignorance in connection with the meaning of the efficiency factor of an electrical machine. This lack of knowledge of the users will be expressed too in meanings of operators about the tolerances of the efficiency classes of electric machines.

The air-gap torque method and the shaft torque method have an anticipated error limit within $0.5 \%$.
The first one needs the no-load point because this point represents the iron losses of the machine. The efficiency of the machine can be calculated by knowing of the total input power and the motor speed. The torque, the total input power and the motor speed must be measured by the second method.

But normally both conditions are very rarely, because the motor shaft is almost connected with the processing machine and the use of torque measurement shafts are very cost intensive in this connection.

\section{Method}

The described method bases on the current circle diagram of an induction machine [2]. At the beginning it must be pointed out that the presented method can use for each type of three phase induction machines such as slip-ring and squirrel cage induction machines.

The shaft torque and subsequently the motor efficiency can be derived from Ossana's circle. In order to get the diagram two points are needed at minimum. The stand still point at rotor speed zero and the no-load point at nearly synchronous speed of the machine. Both points are measured during the start up of the machine, but both points must be corrected due to differences between the real and theoretical conditions of operation.

For the stand still point it means that a few of line periods are used to determine the current amplitude. In order to analyze the point two problems arises. First, when the machine is unloaded or the moment of inertia is too small the analysis of this point is very difficult because the stator current will be damped.

The variation of time of the envelope curve is not an exponential function. It depends on the acceleration torque of the machine and so from the loading conditions of the machine.

Figure 1 shows the stator currents of the phases during an unloaded start up. The different amplitudes of the currents cause by the damped direct currents of each phase. The amplitudes of these currents depend on start up time. 


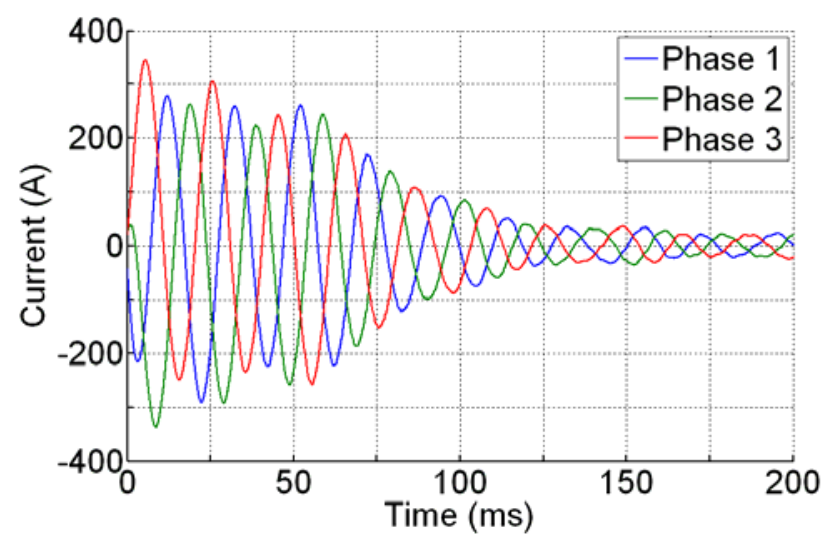

Fig. 1. Time variation of the stator currents during an unloaded start up.

Figure 2 shows the stator currents of the phases during a loaded start up.

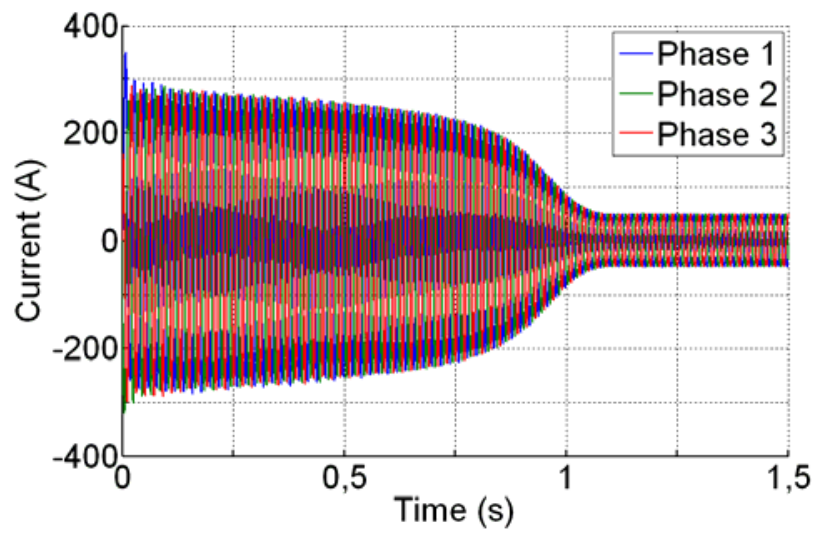

Fig. 2. Phase currents when the motor is a nearly rated load during start up.

Fig. 2 is contrary to Fig. 1. Here are a lot of periods to determine the locked-rotor current of the induction machine.

An useable number of current amplitudes causes by a large moment of inertia of large machines or nearly full load machine. These requirements are always met in practice. The estimation of the stand still current is rather complex. The current during the start up and so the envelope curve is depending on the dynamic behaviour of the motor. Additional there are transient currents during the turn on procedure.

In normal cases, when the motor is loaded near the rated point, the estimation of the transient currents plays a secondary role because the stand still current can be measured after the time when the transient currents are damped to zero. But in the case of an unloaded or only moderate loaded motor, there are not enough periods of the current curve from which the stand still current can be estimated.

To get acceptable results from the current circle diagram of squirrel cage motors it must be presented by two circles because of the saturable leakage inductances and frequency-dependence rotor resistance. The stand still point presents the so-called start up circle. The circle can be constructed by the mean of the diameter [3].

$$
d=\left(U_{1}-I_{m} \cdot X_{1 \sigma}\right) / X_{k}
$$

Equation (1) shows that the diameter depends on the stator voltage, the magnetizing current of the machine and the leakage reactances of the stator and the rotor. It is obviously clear that the magnetizing current during start up will be reduced up to $50 \%$.

The leakage reactances can be derived by the stand still point because the phase angle between the stator voltage and the stator current is known. The second circle will be presented by the no-load point respectively a point between no-load and nominal load.

Because of the awareness of the current curves during start up, the load point at the end of the start up can be corrected in an easy way by the phase angle and the current amplitude. This correction results in the magnetizing current of the motor.

\section{ANALYSIS OF MEASUREMENTS}

\section{A. The stand still current}

The estimation of the stand still current is rather complex. The current during the start up and so the envelope curve is depending on the dynamic behaviour of the motor. Additional there are transient currents during the turn on procedure.

In normal cases, when the motor is loaded near the rated point, the estimation of the transient currents plays a secondary role because the stand still current can be measured after the time when the transient currents are damped to zero. But in the case of an unloaded or only moderate loaded motor, there are not enough periods of the current curve from which the stand still current can be estimated. To estimate the stand still current for all load cases, it is necessary to adjust the phase currents by the transient currents. This can be realized with the space vector of the currents.

\section{B. Magnetizing current}

As mentioned before, two points of the stator current circle diagram can be derived from the start up data. The first point is the stand still current and the second point is the working point.

In order to construct the stator current circle diagram of the motor the magnetizing current is absolutely needed. Because of the circle diameter which can be calculated with equation (1). The magnetizing current can be estimated by the working point, the rating plate data and the equivalent electric circuit.

The working point current can be divided into its real and reactive component. The difference between the calculated reactive part of the working point current and the magnetizing current in equation (1) depends on the loading conditions.

But by the help of the nominal current, the stator resistance and the stator leakage reactance the magnetizing current can be derived from the working point current within acceptable error limits. In addition to this the magnetizing current of standard induction machines are derived from data of test reports. 
By doing this, a maximum possible agreement between the calculated current and the magnetizing current can be found.

\section{Space Vector}

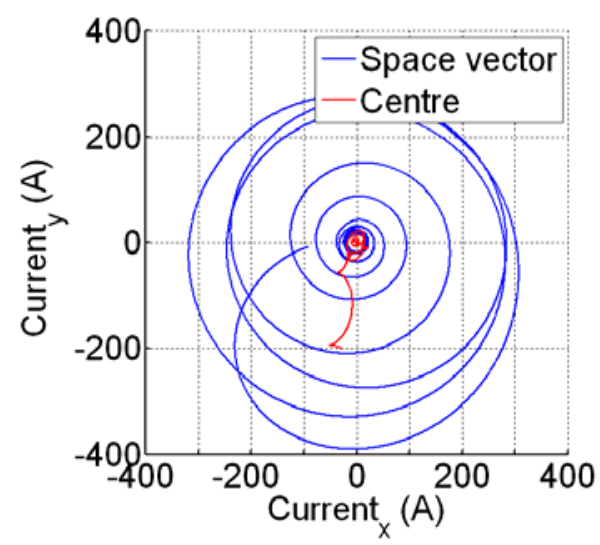

Fig. 3. Space vector of the unloaded start up.

To estimate the stand still current for all load cases, it is necessary to adjust the phase currents by the transient currents. This can be realized with the space vector of the currents.

Normally the centre of rotating current is in origin of the coordinate system. But the transient currents shift the centre out of the origin.

The calculation of the centre and inverse transformation to a three phase system is the base of the estimation of the transient currents. Fig. 3 shows the space vector of an unloaded start up.

The circuit closing of a three phase system effects normally damped D.C. phase currents. The time behaviour of these D.C. currents is given in Fig. 4.

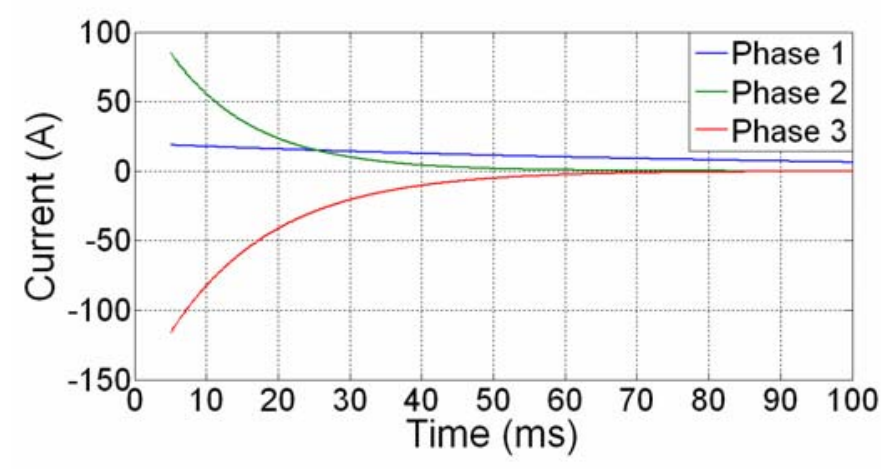

Fig. 4. Time behaviour of the D.C. currents during the unloaded start up.

It must be said, that the determination of the D.C. currents requires a complex optimization algorithm. Fig. 5 shows the estimated currents without the transient currents. This means that the damped direct currents are subtracted.

The calculation of the centre and inverse transformation to a three phase system is the base of the estimation of the transient currents. Fig. 1 shows the space vector of an unloaded start up.

Fig. 5 shows the estimated currents without the transient currents.

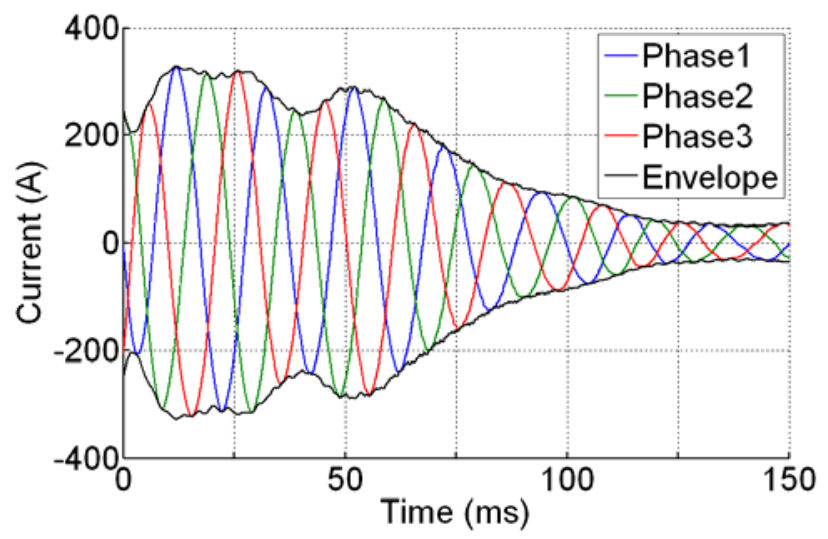

Fig. 5. Phase currents without transient currents.

It is difficult to find the exact centre of the space vector. Consequently the current amplitudes are inaccurate. But if the zero crossings of the current curves are used, the error can be minimized to useful limits.

The phase angle between the supply voltage and the stand still current can be estimated by the difference of the amplitudes between the voltage and the current. Due to the fact that the line-to-line voltage and the phase current are measured the result must be correct by an angle of $30^{\circ}$.

\section{The working point}

The second stationary point which can be used for the described method is the working point. Generally the load at the working point is in the region between 50 to 100 per cent of the nominal load. This point can be analyzed in a very easy way, because there are not the same restrictions as with the starting point.

First, it can be estimated by the amplitude and the phase angle between current and voltage.

Second, it is not necessary to estimate the working point from the start up, because it also can be measured during the working process so long as the load is constant.

\section{E. Stator resistance}

The only measurement beside the start up is the measurement of the resistance.

It can be realize with a dc-measurement. In several cases it is not possible to measure the start up near the motor. You have often a motor control centre were all motors will be switched.

To get the resistance of the stator winding, it is necessary to know the resistance of supply cable between the point of measurement and the motor. Normally the resistance of the supply cable is only a fraction of the stator winding resistance but especially in the case of motors with a high power range the supply cable resistance must be considered. 


\section{CURRENT CIRCLE DIAGRAM}

\section{F. Construction}

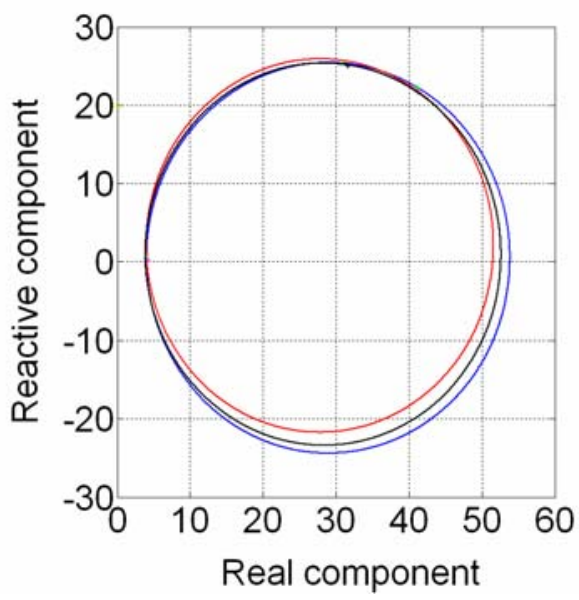

Fig. 6. Current circle diagrams

The current circle diagram can be constructed by various options. First, it can be derived from the equivalent circuit of an induction machine. Second, the current circle diagram is constructed by the method which is described in [4] and [5]. And the end there is the presented method by the help of the stand still point and the no-load point. Fig. 6 shows the current circle diagram constructed by the three different methods. From Fig. 6 can be learned that the sections where the machine works as a motor correspond good. But in the case of an induction machine with a squirrel cage the current circle diagram consists of uncountable circles which depend on the shape of the rotor slots. And without knowing about the shape of the rotor slots these circles, especially the diameters can neither calculated nor estimated from other machine characteristics.

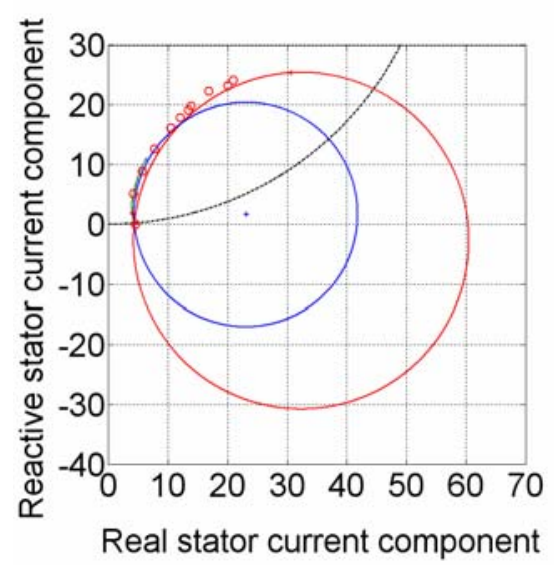

Fig. 7. Current circle diagram of a squirrel cage induction machine.

But from experiments with a lot of standard induction machines can be learned that the motor efficiency can be derived from two circles.

The red circle is constructed by the short circuit point and the no-load point. The small red circles present the measured values. And the blue circle bases on the working point and the no-load point. Fig. 7 shows that the test points correlate up to the nominal point very good with the blue circle.

Only when the machine is overloaded the red circle is needed. So, it can be said, that the motor efficiency can be derived from one common circle. The results obtained by such simplified method have error limit within $0.5 \%$. As mentioned at the beginning only two methods in particular the air-gap torque method and the shaft torque method fulfil the same limits. But they are not practicable because they are too complex.

This circle is constructed by two points, the stand still point and the operation point, it is possible to construct the current circle diagram and get all information for the estimation of the motor efficiency. First, the diameter of the current circle diagram can be calculated with equation (1).

\section{G. Results}

The output power line is defined between the no-load point and the short circuit point. The distance between the circle and this line represents the output power. The input power is given by the amplitude of the current, the input voltage and the phase angle between the voltage and the current. The requirements to the measurement equipment are not very high because of relative small sampling rates. The determination of the magnetizing current must be paid particular attention, because it is in close vicinity to normal operation points. The blue circle in Fig. 7 shows this fact very clearly.

\section{H. Motor shaft torque}

The motor shaft torque can be derived from the current circle diagram, too. But therefore an additional line between the no-load point and the so-called infinite point must be constructed. The infinite point can be found by an additional circle. This circle is given in Fig. 7 by the dotted black line. The radius will be calculated by (2):

$$
r=U_{1} /\left(2 \cdot R_{1}\right)
$$

Normally the speed torque characteristic will be constructed by the use of the current circle diagram. But the evaluation of the speed torque characteristic points out that there are significant differences between the presented current circle diagrams in Fig. 6 despite the fact that the diagrams overlap very well in the range between no-load and overload. Fig. 8 shows the motor shaft torque derived by the current circle diagrams in Fig. 7. The differences in Fig. 8 are because there is not exact speed information measured by a speed counter. The speed information derived from stator current frequencies are not used in this paper. During the start up this method don't yield a result. But in the case of the working point the speed information can be corrected. But that isn't made here because the speed-torque characteristic is not of further interest due to the fact that only the output power, also the multiplication of the speed and torque, is needed to calculate the motor efficiency. 


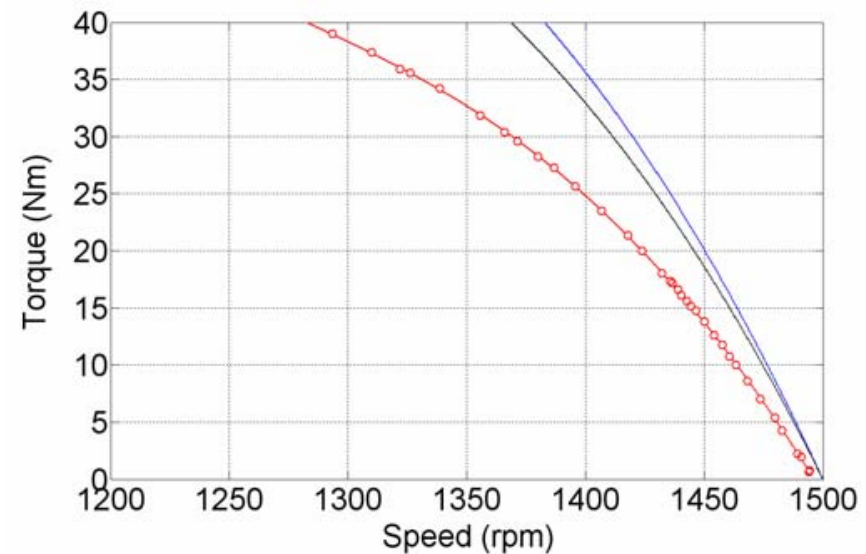

Fig. 8. Speed-torque characteristic (static) of an induction machine.

And in the case of converter fed motor only the currenttorque characteristic of the motor is of interest. This curve is given in Fig.9.

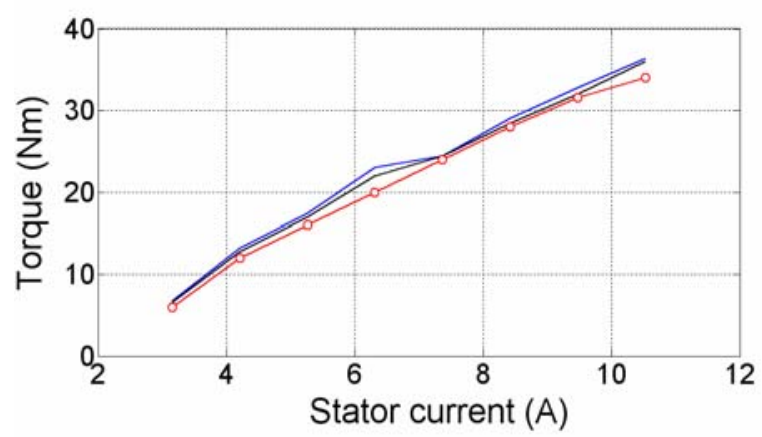

Fig. 9. Stator current-torque characteristic (static) of an induction machine.

\section{Conclusion}

The method to determine the motor efficiency of three phase standard induction machines described in this paper bases on current circle diagram. In spite of its simplicity such as the missing speed and torque information the results are very good. This means that error limit is smaller than $1 \%$. This error is valid when the working point is between 50 and 100 per cent of the nominal power. If it is nearly the un-load point the error will be increase. It must be pointed out that the presented method fulfils the error limit for each type of three phase induction machines such as slip-ring and squirrel cage induction machines. To get acceptable results from the current circle diagram of cage motors it must be presented by two circles because of the saturable leakage inductances and frequency-dependence rotor resistance. This is why the start up of a squirrel cage induction motor must be simulated by non-linear inductances and a variable rotor resistance.

\section{References}

[1] Bin Lu, Th. G. Habelter, A Survey of Efficiency Estimation Methods of In-Service Induction Motors with Consideration of Condition Monitoring Requirements. IEEE 2005, pp 924-932

[2] Th. Bödefeld, H. Sequenz, Elektrische Maschinen, Eine Einführung in die Grundlagen. Wien - New York: Springer - Verlag 1971, pp. 230-236

[3] K.P. Kovács, I. Rács, Elektrische Maschinen, Transiente Vorgänge in Wechselstrommaschinen. 4. Band, Basel/Stuttgart Verlag der Ungarischen Akademie der Wissenschaften Budapest 1959

[4] R. Richter, Elektrische Maschinen, Die Induktionsmaschine. 4. Band, Basel/Stuttgart Verlag Birkhäuser 1954, pp. 40-49

[5] W. Nürnberg, Die Asynchronmaschine. Springer Verlag Berlin- Göttingen-Heidelberg 1952 